\title{
Accelerated sonic fatigue testing methodology for reliability assessments of aircraft structure based on linear load intensification
}

\author{
Chao QU ${ }^{1, a}$, Wenchao HUANG ${ }^{1, b}$ and Dingwen GUO ${ }^{1, c}$ \\ ${ }^{1}$ Aircraft strength research institute, Xi'An 710065, China \\ a email:
}

quchao50@163.com, bemail:huangwenchao@163.com, cemail:guodingwen@163.com

\begin{abstract}
Keywords: Accelerated testing; Sonic fatigue; Random loading; Accelerating factor
\end{abstract}
\begin{abstract}
In term of sonic fatigue, initiation tests are commonly stopped at $10^{8}$ cycles assuming that a fatigue limit exists, whereas feedback experience from in service aircraft has shown that structural elements can fail even after $10^{11}$ cycles. Thus, it is important to explore the gigacycle fatigue domain and an accelerated fatigue testing method which is presented in this paper. A method of accelerated durability testing for failure modes and life cycle based on the results of theory derivation and testing analysis are accomplished. And an acceptable estimation of acceleration factor calculation method based on the stress-lifetime approach ( $S-N$ curve) under narrow-band random acoustic condition is proved. The experimental results show that the application of the reduced time model can be a possible method for accelerated lifetime testing of time-dependent aircraft metal materials.
\end{abstract}

\section{Introduction}

The function of noise on the structure and the object is essentially a kind of dynamic random pressure load of spatial distribution, time-dependent, with a certain frequency distribution characteristics, when the loading quantity is big enough, it would produce a certain stress distribution response causing structural damage on structure, especially when the frequency distribution characteristics of the noise and dynamic characteristics of its function structure coupled with each other. Under the long-term function of such dynamic alternating stress, there would be stress concentration on structure or fatigue cracks until to fatigue fracture on other defective parts, this is known as structural sonic fatigue problems [1].

For military aircraft and civilian aircraft, if only the sonic load on local part is greater than 140 $\mathrm{dB}$, it is need to consider about sonic fatigue problem, the aircraft required sonic fatigue verification before put into use, to prove that the plane will not be damaged due to sonic fatigue within its service life. Considering about the longer service life of aircraft, even if only considering the sonic load period at the hardest state when the aircraft landing and take off, the testing time for verification is very long, then considering to verify matrix fatigue life with sub-sample test, the test time has to be multiplied by dispersion coefficient, so the time will be much longer. Due to overlong of sonic fatigue testing time of aircraft, so you need to adopt the method of accelerated test to shorten the test time.

The theoretical basis of the establishment of acceleration test model is the fatigue life prediction of the structure under random loading. Life prediction can be divided into time domain and frequency domain. As time varies, by obtaining the stress response suffered by the structure under the inspiration of external load, time domain utilizes the stress response and combines Miner's linear cumulative damage rule to conduct life prediction. Time domain analytic method is quite accurate while it requires massive calculation and is quite complicated in practical use. Frequency domain analytic method utilizes frequency-response function of the structure, also named transfer function, to obtain the power spectral density(PSD) of stress response in the frequency domain, then to obtain the all-order spectral moment of PSD through the principles of statistics to obtain probability density function of the stress peaks(PDF), thus predicting the fatigue life by combining the fatigue damage model. The frequency domain possesses less calculated quantity and is more 
adopted in engineering practice. Based on the research perspective of fatigue life prediction of frequency domain, Roberto Tovo[2-3] has summarized the fatigue damage prediction method under the broadband loading spectrum together with other experts and conducted researches on the relationship between the fatigue damage and different counting methods of loading in frequency domain. Besides, he has also proposed a kind of fatigue life prediction method which is applicable for a stationary zero-mean broad-band Gaussian random process.

The propose of accelerated test method is finding the relationship between the life of the structure under diverse random loading. Scholars both at home and abroad had carried a lot of studies on fatigue accelerated test. Allegri [4]studied the fatigue hardening coefficient model applicable for stationary narrow-band random process. J.M.Hu[5]derived a simple structural component vibratory fatigue acceleration equation with material fatigue service life method based on narrow-band Gaussian random signal. J.J.Xiong[6]establish a load history generation approach for full-scale accelerated fatigue tests.Chen Sheng-jun[7] proposed a fatigue hardening coefficient model based on general scale method applicable for stationary broad-band random process. Wang Dong-mei [8] discussed the calculation method of random vibration test acceleration factor under narrowband and broadband random load. However, most of these studies were aimed at the accelerated test method for vibration fatigue problems, and cannot be directly applied to sonic fatigue acceleration test. It proposed a sonic fatigue acceleration test method for aircraft structure in "Sonic fatigue design manual"[9], but no applicable conditions of the method, and made a detailed introduction of the selection method of accelerating factor.

Based on the random vibration fatigue acceleration test method, this paper deduced an acceleration test method suitable for sonic fatigue, also analyzed its applied conditions and selection method of accelerating factor, and verified the rationality of this accelerated test method by test.

\section{Derivation of sonic fatigue acceleration test equation}

Suppose the $S-N$ curvilinear equation of a certain structural acoustic fatigue as[10]

$\lg S=A+B \times \lg N_{S}$

The accumulative damage of components under a certain stress level as:

$$
D=\int_{0}^{\infty} \frac{n_{s}}{N_{s}} d s
$$

In the equation, $n_{s}$ behaves as the actual cycle number when the stress amplitude comes to and can be indicated as the probability density function of the stress peaks $P(s) ; N_{s}$ serves as the damage cycle number when the stress amplitude comes to and can be established by $S-N$ curvilinear equation.

$$
n_{s}=E(p) T p(s)
$$

In the equation, $E(p)$ is the expected number of the zero upcrossing rate which can be considered as the expected number of stress cycle per unit time for a narrow-band random process serves as the expected value which can randomly respond to the signal peak frequency, $T$ serves as the action time which also can randomly respond, $P(s)$ serves as the probability density function of stress peak. The following equation can be obtained when substituting equation (1) and (3) into equation (2):

$$
D=E(p) T \int_{0}^{\infty} 10^{\frac{A-\lg s}{B}} p(s) d s
$$

Under general condition, when $D=1$ and the structure suffers damage, the lifetime when the components are damaged can be obtained:

$$
T=\frac{1}{E(p) \int_{0}^{\infty} 10^{\frac{A-\lg s}{B}} p(s) d s}
$$

The $n$th spectrum moment of a PSD can be defined as: 


$$
m_{n}=\int_{-\infty}^{+\infty} f^{n} G(f) d f
$$

In the equation, $f$ serves as the frequency and $G(f)$ serves as the power spectral density. As for a steady-state gaussian random process, its expected value of peak frequency $E_{P}=\left(m_{4} / m_{2}\right)^{1 / 2}$, the average crossing rate $E_{0}=\left(m_{2} / m_{0}\right)^{1 / 2}$, the irregular factor $\gamma=E_{p} / E_{0}$, the broadband coefficient $\varepsilon=\left(1-\gamma^{2}\right)^{1 / 2}$.

The literature [11] has compared the accuracy of rain-flow amplitude distribution of stress cycle of four kinds of probability density function of the stress peaks models on different spectral-width coefficient, and assigned various applicable domain of probability density function of the stress peaks. As for narrow-band random process when the spectral-width coefficient $\varepsilon<0.3$, the stress amplitude is considered to be distributed approximately along Rayleigh. Therefore, as for the narrow-band random process, the peak probability density function $P(s)$ can be indicated as:

$$
P(S)=\frac{S}{\sigma^{2}} \exp \left(-\frac{S^{2}}{2 \sigma^{2}}\right)
$$

As for the steady-state gaussian random process, the amplitude probability density function stays consistent with the peak probability density function, besides, $E_{p}=E_{0}$. Suppose the original testing time of a certain structure as $T_{r}$, and the testing time after loading linear strain-hardening as $T_{s}$. The definition of the accelerated factor as:

$k=T_{r} / T_{s}$

The following equation can be obtained when substituting equation (2) and (5) into equation (8):

$$
k=\frac{T_{r}}{T_{s}}=\frac{E(p)_{s} \int_{0}^{\infty} s_{s}^{10^{\frac{A-\lg s}{B}}} p(s)_{s} d s}{E(p)_{r} \int_{0}^{\infty} s_{r}^{10^{\frac{A-\lg s}{B}}} p(s)_{r} d s}
$$

As both the shape and the frequency domain of the loading power spectral density before and after the accelerated test stay invariable and the value of actual accelerated power spectral density has been magnified, the expected peak frequency $E(p)$ and the peak probability density function $P(s)$ before or after the acceleration will stay invariable. The equation(9)can be simplified as:

$$
k=\frac{T_{r}}{T_{s}}=10^{-\frac{1}{B} \lg \left(\frac{S_{s}}{S_{r}}\right)}
$$

As for the acceleration test of acoustic fatigue, the most different aspect from the acceleration test of vibration fatigue is that the load is applied by way of sound. The magnitude needs to be described by sound pressure level, while vibration test adopts acceleration to describe the load magnitude. Suppose the sound pressure level arousing $S_{r}$ as $S P L_{r}$, the corresponding sound pressure is $P_{r}$, and the lifetime of the structure under the $S P L_{r}$ sound pressure level is $T_{r}$; Suppose the sound pressure level arousing $S_{t}$ as $S P L_{t}$, the corresponding sound pressure is $P_{t}$, and the lifetime of the structure under the $S P L_{t}$ sound pressure level is $T_{t}$; Suppose $\triangle S P L=S P L_{t}-S P L_{r}$. Suppose the structural response stays inside the linear response domain before and after the acceleration, then

$$
\frac{S_{t}}{S_{r}}=\frac{P_{t}}{P_{r}}
$$

The equation (10) can be indicated as:

$k=\frac{T_{r}}{T_{s}}=10^{-\frac{1}{B} \lg \left(\frac{P_{s}}{P_{r}}\right)}$

The following equation can be obtained according to the definition of the sound pressure level:

$$
\Delta S P L=20 \lg \left(\frac{P_{r}}{P_{0}}\right)-20 \lg \left(\frac{P_{s}}{P_{0}}\right)=20 \lg \left(\frac{P_{r}}{P_{s}}\right)
$$

The following equation can be obtained by equation(12) and (13): 


$$
k=\frac{T_{r}}{T_{s}}=10^{-\frac{1}{B} \times \frac{\Delta S P L}{20}}
$$

The equation is supposed to obtain the equation of life proportion before and after the acceleration when the difference of sound pressure level before and after the acceleration is known, also named the equation of accelerated factor. Which can be seen from equation(14) is that the accelerated factor is the function of parameter $B$ of structural material. $B$ serves as the slope of log-log coordinate of $S-N$ curve structure. If both sides of equation(12) are rounded off, the following equation can be obtained:

$$
\lg P_{r}-\lg P_{s}=B \times\left(\lg T_{r}-\lg T_{s}\right)
$$

Which can be seen from equation(15) is that the load sound pressure is linearly correlated with the structural fatigue life under the log-log coordinate, and the slope serves as the slope under the log-log coordinate of $S-N$ curve structural acoustic fatigue.

\section{Condition of sonic fatigue acceleration test formula}

Using linear strengthening load spectrum for acceleration test, the several conditions should be satisfied[8]:

a) Material is linear elastic.

b) No geometric nonlinearity exists in the structure (only small stress change).

c) Stationary gaussian process of random excitation at zero-mean.

For condition a),b), can be understood as the sound pressure needs of test load and stress response should be linear relationship. For the condition c), the gaussian process indicates observing random process at any time, if the probability distribution of random variables is satisfied the gaussian distribution, the random process is referred as the gaussian process, general sonic fatigue test can meet this condition.

Considering the sonic fatigue acceleration test formula is based on the theory of Miner linear cumulative damage, so before and after the acceleration, material can't enter into the plastic area. Sonic fatigue acceleration test formula, moreover, is based on the constant sound load spectrum to change the sound pressure level for acceleration, if the main stress response peak frequency of the structure before and after acceleration was changed a lot, although sound load remains the same, but the response frequency characteristic of the structure was significantly changed, so before and after acceleration, the main stress response peak frequency of the structure cannot have changes. Bu the way, it can be find that the equation(14) turned out to be correct with differend probability density function of the stress peaks $P(s)$.

The condition of sonic fatigue acceleration test method can be concluded as:

a) The load sound pressure and the stress response of the specimen on the same test piece should be a linear relationship before and after acceleration.

b) The main stress response frequency of the same test piece cannot be changed before and after acceleration.

\section{Verification of sonic fatigue acceleration test formula}

To verify the proposed sonic fatigue acceleration test formula by this test. The test piece is an aluminum alloy 7075 -T6 rectangular plate, the size of it is $340 \mathrm{~mm}^{*} 250 \mathrm{~mm} * 1.5 \mathrm{~mm}$. The plate around is fixed on the fixture by rivets. Install the test piece on the side wall of the traveling-wave tube for test, with the method of grazing incidence incentives, to make the direction of sound wave propagation is parallel to the specimen surface, the installation of test piece of traveling-wave tube is shown in Figure 1. 


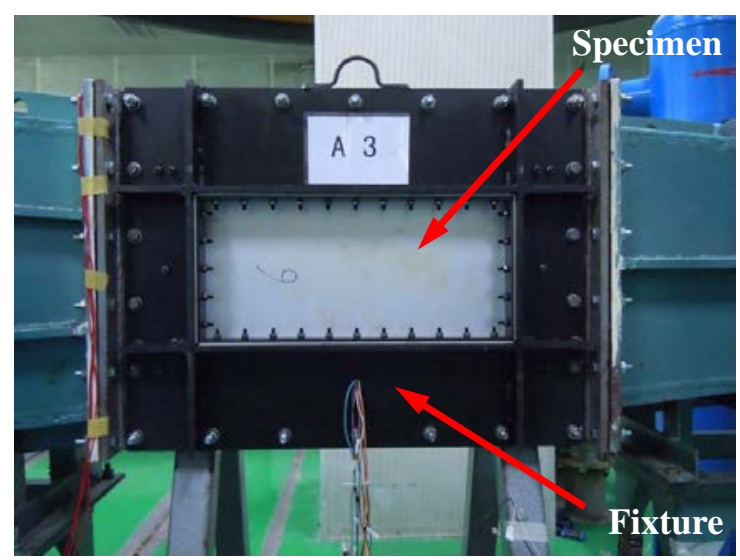

Fig.1. The specimen and clamping fixture

24 test pieces are divided into 8 different sound load magnitude groups, sonic fatigue destructive test is carried out by loading $1 / 3$ octave narrowband flat spectrum with $160 \mathrm{~Hz}$ as the center frequency. Pasting stress rosette at the middle of the long sides of the test piece for stress measurement, when there is visible cracks on the surface of the test piece by visual inspection, the test piece can be viewed as damage, record its fatigue life and stress measurements, using the least square method for data fitting. The median acoustic fatigue life $S_{r m s}-N$ curve of aluminium alloy 7075-T6 material with $50 \%$ survival rate is given in Figure 2, its expression formula is $\lg S_{r m s}=2.943-0.199 \lg N$, where $S_{r m s}$ is the maximum $R M S$ principal stress, $N$ is the sonic fatigue life. The slope of curve $B=-0.199$, association coefficient $R=-0.861$ 。

Then substituting $B=-0.199$ into formula (14), it can established the sonic fatigue acceleration test formula of aluminum alloy 7075 - T6 material. This formula expressed, for the same structure, in the case of constant sonic load spectrum, the relationship between the loaded total sound pressure and its sonic fatigue life, obviously, accelerating factor associated with $B$. It can also make the relation curve of the total sound pressure and its sonic fatigue life from the test result, on double logarithmic coordinate, using least square method for data fitting of the load sound pressure and sonic fatigue life of 24 test pieces, the obtained curve slope is - 0.217 , compared the obtained curve slope with the given curve slope from formula (15) which is -0.199 , as shown in Figure 3.

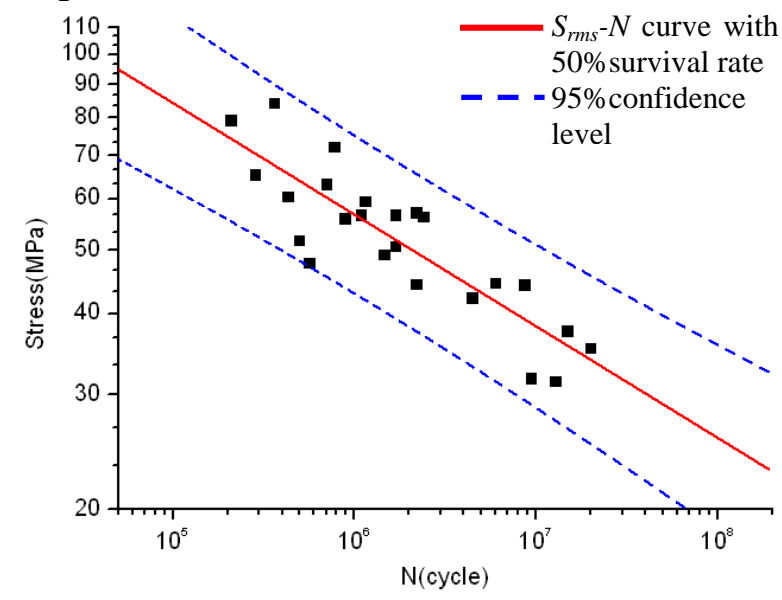

Fig.2. Noise fatigue random $S_{r m s}-N$ curve of 7075-T6 


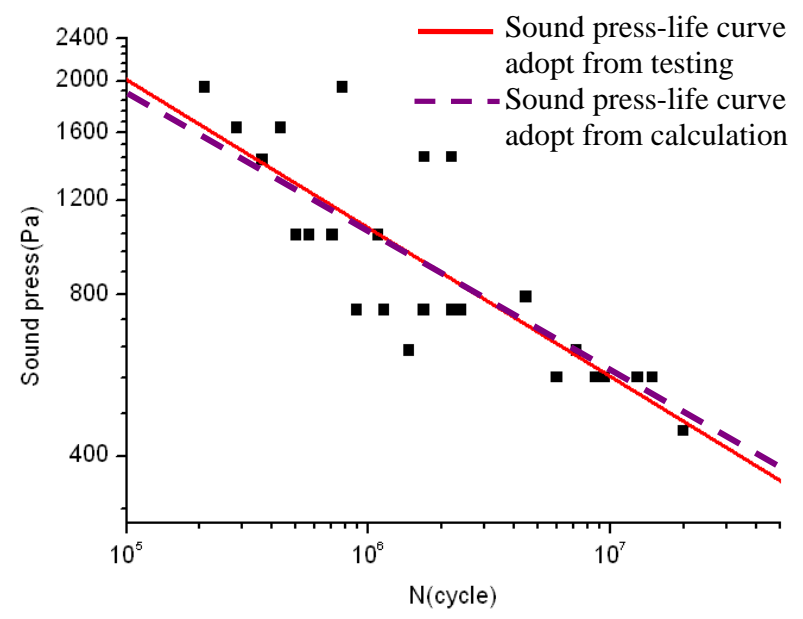

Fig.3. Comparison of the testing result and calculated result of acceleration test formula

It can be seen from figure 3, the obtained slope of sonic fatigue acceleration test curve is relatively close to the given slope by formula (15), it is proved the rationality of the accelerated test formula (14). As can be seen from the result, absolute value of the slope of the sonic fatigue acceleration test obtained by test is larger, so if using formula (14) for sonic fatigue acceleration test, as increasing the sound pressure level, the designed test time is greater than its verification time, so the acceleration test process is conservative.

For sonic fatigue acceleration test, in addition to the required two conditions proposed in this paper, there also should try to choose the $S-N$ curve slope of original life verify and accelerated life test as the parameters of formula (14). This is why for general aviation aluminum alloy material, its ultrahigh cycle $S-N$ curve slope is relatively changed more than the lower life range, as the absolute value of slop is gradually decreased as the increase of the lifetime, if choosing lower lifetime span of $S-N$ curve slope for ultrahigh cycle fatigue acceleration test, the results may be too conservative.

\section{Conclusion}

This paper proposed a sonic fatigue acceleration test method can be used in typical structure of aircraft, and proved the rationality of this acceleration test method through experiment. The conclusions can be drawn as follows:

a) For the structure of aircraft, it can adopts linear strengthening load spectrum method for sonic fatigue test acceleration.

b) The condition of the proposed acceleration test formula: before and after acceleration, the relation of sound pressure of sound load and stress response of test piece should be linear, and the main stress response frequency of the test piece can not be changed.

c) The sonic fatigue accelerated factor is only associated with the slope of sonic fatigue $S-N$ curve of the material in log-log coordinate figure.

It obtained the median sonic fatigue $S_{r m s}-N$ curve of aluminium alloy 7075-T6 material with $50 \%$ survival rate by experiment.

\section{References}

[1] Satoshi Kagami,Tomonobu Kitagawa,Koichi Nishiwaki,Tomomichi Sugihara,Masayuki Inaba,Hirochika Inoue. A Fast Dynamically Equilibrated Walking Trajectory Generation Method of Humanoid Robot [J], 2002.

[2] HIROKIK, MINORUA, YASUOK, ET al.RoboCup: a challenge problem for AI and robotics. HirokiK. RoboCup-97: Robot Soccer World Cup [C]. Berlin: Springer, 1998.38-43.

[3] Zhizhong Yin. Application of FPGA control DC motor servo system [J]. Inner Mongolia Science and Technology and Economy, 2008177 (23) 101-103.

[4] Joerg Christian Wolf, Phil Hall, Paul Robinson, Phil Culverhouse. Bioloid based Humanoid 
Soccer Robot Design, 2007.

[5] Wu Chuan-yu, He Lei-ying, Design and Realization of Instructional RPPR-Robot, Research and Exploration in Laboratory.2007, 26(10)

[1] Diyi Tang, Wenlan Li, Weiyang Qiao. The elaments of aircraft noise [M]. Xi'an: Northwestern Polytechncical University Press, 1995. (Ch).

[2] Roberto Tovo. Cycle distribution and fatigue damage under broad-band random loading[J]. International Journal of Fatigue, 2002, 24: 1137-1147.

[3] D. Benasciutti, R. Tovo. Spectral methods for lifetime prediction under wide-band stationary random processes[J]. International Journal of Fatigue, 2005, 27: 867-877.

[4] Allegri G, Zhang X. On the Inverse Power Laws for Accelerated Random Gatigue Testing [J]. International Journal of Fatigue, 2008, 30:967-977.

[5] J.M.Hu, D.Barker, A.Dasgupta. Role of Failure-mechanism Identification in Accelerated Testing, Proceedings Annual Reliability and Maintainability Symposium, 1992.

[6] J.J. Xiong, R.A. Shenoi. A load history generation approach for full-scal accelerated fatigue tests[J]. Engineering Fracture Mechanics 2008, 75:3226 - 3243.

[7] Junsheng Cheng, Yi Yuan, Zhentao Yu, Hui Yuan. Analysis of Vibration Fatigue Strength Coefficient Based on General Scaling Law [J].Noise And Vibration Control,2014, 34(1): 47-51. (Ch).

[8] Dong-mei WANG, Jin-song XIE. Acceleration Factor Calculation Method of Random Vibration Test [J].Environment Technology. 2010,04(2):47-51. (Ch).

[9] Qihang YAO, Xueqin YANG. The Guideline manual of sonic fatigue for aircraft structures [M]. Beijing: Aviation Industry Press, 1998. (Ch).

[10] NATO Standardisation Agency, The Ten Step Method for Evaluating The Ability of Materiel to Meet Extended Life Requirements And Role And Deployment Changes, AECTP600(edition 2), Apr 2007.

[11] YANG Wan-jun, SHI Rong-ming Research on Stress Amplitude Distribution of Random Vibration. [J] Machine Design and Research。2011, 27: 16-20. 